\title{
CHARACTERS OF $p$-GROUPS
}

\author{
CHARLES E. FORD
}

(Communicated by Bhama Srinivasan)

\begin{abstract}
The following theorem is proved for any prime $p$ :
Every irreducible rational represention of a finite p-group is the difference of two transitive permutation representations.

Also given are two useful results about representations of $p$-groups, which are known to experts in the field.
\end{abstract}

1. Statement of results. Presented here are three results about characters of finite $p$-groups. The first two are probably known to people familiar with the subject. The first follows directly from a theorem of Solomon [7, p. 156, Theorem 4] and the second from a generalization of this in Feit [2, p. 73, 14.3]. Since they do not seem to have been stated and proved explicitly our purpose in doing so here is to make them available to a wider audience.

The third result generalizes a theorem of Graeme Segal [6] that may not be well known among group theorists. As Segal states in his paper, Feit has observed that this theorem may be proved using the result above. We present such a proof here and obtain the following result.

THEOREM. An irreducible rational representation of a finite p-group, $p$ a prime, is the difference of two transitive permutation representations.

Let $Q$ denote the rational field and $Q G$ the rational group algebra of a finite group $G$. Let $p$ be a prime number. Gal denotes a Galois group. $\left[X_{2}: X_{1}\right]$ denotes the index of $X_{1}$ in $X_{2}$ for either fields or groups. Absolute value bars denote order. All groups are finite here.

THEOREM 1. Let $G$ be a p-group and $\chi$ an irreducible complex character of $G$. Then one of the following holds:

(i) There exists a linear character $\lambda$ on a subgroup $H$ of $G$ which induces $\chi$ and generates the same field as $\chi$; that is $\lambda^{G}=\chi$ and $Q(\lambda)=Q(\chi)$.

(ii) $p=2$ and there exist subgroups $H<K$ in $G$ with $[K: H]=2$ and a linear character $\lambda$ of $H$ such that with $\varsigma=\lambda^{K},[Q(\lambda): Q(\varsigma)]=2, \varsigma^{G}=\chi$, and $Q(\varsigma)=Q(\chi)$.

THEOREM 2. Let $G$ be a p-group and $\chi$ an irreducible complex character of $G$ for which the Schur index $m_{Q}(\chi)=2$. Then $G$ contains a generalized quaternion section. More specifically there is an irreducible character $\zeta$ on a subgroup $K$ of $G$

Received by the editors August 20, 1986. Presented at the San Antonio Meeting of the AMS on January 22, 1987.

1980 Mathematics Subject Classification (1985 Revision). Primary 20C15.

Key words and phrases. Representation, $p$-group, Schur index.

(C) 1987 American Mathematical Society $0002-9939 / 87 \$ 1.00+\$ .25$ per page 
for which $\varsigma^{G}=\chi, Q(\varsigma)=Q(\chi)$ is a subfield of the real field, and the image of $K$ under a representation affording $\varsigma$ is a generalized quaternion group.

THEOREM 3. Let $G$ be a p-group and $\theta$ the rational valued character of $G$ obtained as the sum of the algebraic conjugates of an irreducible complex character $\chi$. Then there exist subgroups $G_{1}<G_{2}$ in $G$ with $\left[G_{2}: G_{1}\right]=p$ such that $\theta=$ $(1 / m)\left(\lambda_{1}^{G}-\lambda_{2}^{G}\right)$ where $\lambda_{i}$ is the principal character on $G_{i}$ and $m=m_{Q}(\chi)$ ( $m$ is 1 if $p$ is odd and 1 or 2 if $p=2$ ). In particular each irreducible rational representation of $G$ is the difference of two transitive permutation representations.

The last statement of the theorem follows with multiplication by $m$ from the fact $[2$, p. $62,11.4]$ that the characters of irreducible $Q G$ modules are all of the form $m_{Q}(\chi) \sum \chi^{\sigma}$, summed over the conjugates of irreducible complex characters $\chi$. Segal's theorem states that every such character is an integral linear combination of permutation characters.

The Schur index $m_{Q}(\chi)$ can be defined as the smallest integral multiple of the rational valued character $\theta=\sum \chi^{\sigma}$ which is afforded by a $Q G$ module. It can be shown to divide the smallest multiple of $\theta$ which can be expressed as an integral linear combination of permutation characters. See for example the argument of Feit in [1, p. 294].

It is natural to consider here the Artin induction theorem [2, p. 69, 13.2] which asserts that for every rational valued character of a group, some integral multiple is an integral linear combination of characters induced from principal characters of cyclic subgroups. One could call the smallest such multiple the Artin order of the character. T. Y. Lam [5] has defined the least common multiple of such orders to be the Artin exponent of the group and has shown [5, p. 110, Theorem 6.3] that even for $p$-groups, these orders can be quite large. One could define the order more generally by removing the restriction that induction be from cyclic subgroups and allow any subgroup. Segal's theorem asserts that for $p$-groups, this order is the Schur index.

2. Proofs of Theorems 1 and 2. We begin by restating Feit's result in the special case of a $p$-group.

THEOREM A. Let $G$ be a finite p-group and $\chi$ a nonlinear irreducible faithful character of $G$. Suppose $G$ does not contain a cyclic subgroup of index 2 . Then there exists a character $\mu$ on a subgroup $F$ of index $p$ in $G$ for which $\mu^{G}=\chi$ and $Q(\mu)=Q(\chi)$.

Observe that $\mu$ must be irreducible since it induces an irreducible character. Consider now the exceptional case in this theorem, when $G$ is a 2-group which contains a cyclic subgroup $H$ of index 2. If $G$ is abelian then all the irreducible characters are linear. If $G$ is nonabelian there are four possibilities: generalized quaternion, dihedral, semidihedral, and $M_{n}(2)$ (see Gorenstein [4, p. 193, Theorem 4.4]).

For all such groups a faithful irreducible character $\varsigma$ has degree 2 and is induced from a faithful character $\lambda$ on the cyclic subgroup $H$, according to Feit $[\mathbf{2}$, p. 63 , 11.6]. It is not difficult to see that $[Q(\lambda): Q(\varsigma)]=2$. If $G$ is not generalized quaternion there is an element of order 2 lying outside $H$. For such $G$ Feit has 
shown that $M_{Q}(\varsigma)=1[2$, p. 64, 11.7], while for a generalized quaternion group $m_{Q}(\varsigma)=2[2$, p. $64,11.8]$.

We now proceed to a proof of Theorem 1 . Thus $G$ is a $p$-group with irreducible complex character $\chi$. If $\chi$ is linear the theorem is trivially true, so assume $\chi$ is not linear. Suppose first that $G$ has a cyclic normal subgroup of index 2 . Then our discussion above shows alternative (ii) holds with $K=G$ and $\varsigma=\chi$ and the theorem is proved in this case. Next suppose that $G$ does not contain a cyclic subgroup of index 2. Then Theorem A gives a subgroup $F$ of index $p$ with an irreducible character $\mu$ for which $\mu^{G}=\chi$ and $Q(\mu)=Q(\chi)$. Although $\mu$ need not be faithful, by induction on the order of the group we assume the theorem holds for the image of $F$ under a representation affording $\mu$. Whichever alternative of the theorem holds for this group will also, by transitivity of the induction of characters, hold for $G$ and Theorem 1 is proved.

Now we prove Theorem 2. Apply Theorem 1 to a group $G$ and character $\chi$ satisfying the hypotheses of Theorem 2. Were alternative (i) to hold then $\lambda^{G}=\chi$ would give a representation affording $\chi$ in the field of values of $\lambda$, and $Q(\lambda)=Q(\chi)$ would give $m_{Q}(\chi)=m_{Q}(\lambda)=1$, contrary to assumption. Thus alternative (ii) must hold. Again $Q(\varsigma)=Q(\chi)$ gives that $m_{Q}(\varsigma)=m_{Q}(\chi)$, so our hypotheses imply that $m_{Q}(\varsigma)=2$. Consider the images of $H$ and $K$ under a representation affording $\varsigma$. It is not difficult to see that $\lambda$ and $\varsigma$ have the same kernel, and since $\lambda$ is linear, the image of $H$ is a cyclic subgroup of index 2 in the image of $K$. From our discussion earlier, the fact that $m_{Q}(\varsigma)=2$ forces this image to be a generalized quaternion group. The assertion that $Q(\varsigma)$ is a real field follows from the fact that $\varsigma$ vanishes off $h$ and on $H$ has value $\lambda+\bar{\lambda}$ (see [2, p. 63, 11.6]). Theorem 2 is proved.

Proof of Theorem 3. We need the following Lemma for Theorem 3.

LEMMA. Let $G$ be a cyclic $p$-group of order $p^{n} \geq p$. Let $\lambda$ be a faithful linear character on $G$. Let $\mu=\sum \lambda^{\sigma}$ be the sum of all the conjugates of $\lambda$ under the action of $\operatorname{Gal}(Q(\lambda): Q)$. Then $\mu=\lambda_{1}^{G}-\lambda_{2}^{G}$, where $\lambda_{1}$ is the principal character on the identity subgroup, and $\lambda_{2}$ is the principal character on the subgroup $Z$ of order $p$.

PROOF. The order of the Galois group is $p^{n}-p^{n-1}$. Therefore $\mu(1)=p^{n}-p^{n-1}$. Let $g$ in $G$ have order $p^{k}$. Then $\mu(g)$ is $p^{n-k}$ multiplied by the sum of the distinct conjugates of a primitive $p^{k}$ root of unity. For $k>1$ this sum is zero and for $k=1$ it is -1 , as can be seen by investigating the cyclotomic polynomial. Thus $\mu(g)=-p^{n-1}, g \neq 1$, for $g$ in $Z$ and $\mu(g)=0$ for $g$ outside $Z$. The Lemma now follows because $\lambda_{1}^{G}$ takes value $p^{n}$ on the identity and vanishes elsewhere, and $\lambda_{2}^{G}$ takes value $p^{n-1}$ on $Z$ and vanishes elsewhere.

We now prove Theorem 3. We have $\theta=\sum \chi^{\sigma}$ with the sum taken over $\operatorname{Gal}(Q(\chi): Q)$. Suppose alternative (i) of Theorem 1 holds for $G$ and $\chi$. Then $\chi=\lambda^{G}$ for a linear character $\lambda$ on a subgroup $H$. Let $G_{1}$ be the kernel of $\lambda$ and $G_{2}$ the subgroup of $H$ with $\left[G_{2}: G_{1}\right]=p$. Let $\lambda_{i}$ be the principal character of $G_{i}$. We now apply the Lemma to the image of $H$ under $\lambda$, using the fact that all the algebraic conjugates of $\lambda$ have the same kernel, and conclude that $\sum \lambda^{\sigma}=\lambda_{1}^{H}-\lambda_{2}^{H}$. 
But since conjugation and induction commute and are both linear

$$
\theta=\sum \chi^{\sigma}=\left(\sum \lambda^{\sigma}\right)^{G}=\left(\lambda_{1}^{H}-\lambda_{2}^{H}\right)^{G}=\lambda_{1}^{G}-\lambda_{2}^{G}
$$

The two summations are over the same Galois group because $Q(\lambda)=Q(\chi)$. This completes the proof for alternative (i).

Now suppose alternative (ii) holds. Then there exist subgroups $H$ and $K$ in $G$ with $[K: H]=2$ and a linear character $\lambda$ of $H$ such that $\lambda^{K}=\varsigma, \varsigma^{G}=\chi$, $[Q(\lambda): Q(\varsigma)]=2$, and $Q(\varsigma)=Q(\chi)$. In this case there is a unique correspondence between conjugates of $\zeta$ and conjugates of $\chi$ and we have

$$
\theta=\sum \chi^{\nu}=\left(\sum \varsigma^{\nu}\right)^{G}
$$

where the sum is over $\operatorname{Gal}(Q(\varsigma): Q)$. However $\left.\varsigma\right|_{H}=\lambda+\lambda^{\tau}$ where $\tau$ generates $\operatorname{Gal}(Q(\lambda): Q(\varsigma))$. Therefore $\left.\sum \varsigma^{\nu}\right|_{H}=\sum \lambda^{\sigma}$ where the latter sum is over $\operatorname{Gal}(Q(\lambda): Q)$.

Since $\zeta$ is irreducible the image of $K$ under a representation affording $\zeta$ is one of the four nonabelian groups discussed earlier. If $m_{Q}(\chi)=2$ this group is generalized quaternion. We consider this case first. If we let $G_{1}$ be the kernel of $\lambda$ and $G_{2}$ the subgroup of $H$ with $\left[G_{2}: G_{1}\right]=2$ as we did in alternative (i) above, then the Lemma implies that

$$
\left.\sum \varsigma^{\nu}\right|_{H}=\sum \lambda^{\sigma}=\lambda_{1}^{H}-\lambda_{2}^{H}
$$

If we now induce further up to $K$ we claim that $\lambda_{1}^{K}-\lambda_{2}^{K}=2 \sum \varsigma^{\nu}$. This follows with Frobenius reciprocity

$$
\left(\varsigma^{\nu}, \lambda_{1}^{K}-\lambda_{2}^{K}\right)=\left(\left.\varsigma^{\nu}\right|_{H}, \lambda_{1}^{H}-\lambda_{2}^{H}\right)=2
$$

because each $\left.\varsigma^{\nu}\right|_{H}=\lambda^{\nu}+\lambda^{\nu \tau}$ contributes two linear characters to $\sum \lambda^{\sigma}=\lambda_{1}^{H}-\lambda_{2}^{H}$. Induction from $K$ to $G$ gives

$$
\lambda_{1}^{G}-\lambda_{2}^{G}=2\left(\sum \varsigma^{\nu}\right)^{G}=2 \theta
$$

Dividing by $m=2$ proves the theorem in this case.

In the remaining cases, $m_{Q}(\chi)=1$ and the image of $K$ under $\zeta$ is either dihedral, semidihedral, or of type $M_{n}(2)$. Unlike the generalized quaternion group, each of these is a semidirect product of a cyclic group, the image of $H$, with a group of order 2. We will identify $H$ and $K$ with their images under a representation affording $\varsigma$ and assume $K=H B$ where $H$ is cyclic of order $2^{n}$ and $B$ has order 2 . In the generalized quaternion case we defined $\lambda_{1}$ and $\lambda_{2}$ on subgroups of $H$. Eventually we were forced to induce from $H$ to $K$, which produced the coefficient 2 . We will avoid this in these remaining cases by including $B$ in the kernels of $\lambda_{1}$ and $\lambda_{2}$. Let $G_{1}$ be the subgroup whose image is $B$ and $G_{2}$ the subgroup with image $B Z$. Let $\lambda_{i}$ be the principal character of $G_{i}$.

Our goal is to prove that $\sum \varsigma^{\nu}=\lambda_{1}^{K}-\lambda_{2}^{K}$, for then the theorem will follow by inducing these characters from $K$ to $G$, as we did in alternative (i). It follows from the Mackey subgroup theorem $\left[1\right.$, p. 324, 44.2] that $\left.\lambda_{1}^{K}\right|_{H}=\left(\left.\lambda_{1}\right|_{H \cap B}\right)^{H}=\pi_{1}^{H}$ where $\pi_{1}$ is the principal character on the identity subgroup. Similarly $\left.\lambda_{2}^{K}\right|_{H}=\pi_{2}^{H}$ 
where $\pi_{2}$ is the principal character on $Z$. According to the Lemma, $\sum \lambda^{\sigma}=$ $\pi_{1}^{H}-\pi_{2}^{H}$. Thus we have

$$
\left.\sum \varsigma^{\nu}\right|_{H}=\sum \lambda^{\sigma}=\pi_{1}^{H}-\pi_{2}^{H}=\left.\left(\lambda_{1}^{K}-\lambda_{2}^{K}\right)\right|_{H} .
$$

This shows that the equation $\sum \varsigma^{\nu}=\lambda_{1}^{K}-\lambda_{2}^{K}$ holds on $H$. We must now show it holds on $K \backslash H$. Since $\varsigma$ vanishes off $H$, and consequently so does $\sum \varsigma^{\nu}$, we must show that $\lambda_{1}^{K}-\lambda_{2}^{K}$ also vanishes off $H$. This will require a separate analysis of each case. Let $h, b$, and $z$ be generators of $H, B$, and $Z$ respectively. Then $|h|=2^{n},|b|=|z|=2$, and $z=h^{2^{n-1}}$.

Consider first the dihedral case where $b^{-1} h b=h^{-1}$. A calculation shows that $h^{-1} b h=b h^{2}$. Thus outside $H$ there are two conjugacy classes, one containing products of $b$ with the odd powers of $h$ and the other $b$ with the even powers of $h$, including $b$ and $b z$. Now $\lambda_{1}^{K}$ is induced from $B$ and vanishes on the conjugacy class not containing $b$. The centralizer $C(b)$ of $b$ is $B Z$. It follows that $\lambda_{1}^{K}(b)=$ $|C(b)| /|b|=2$. Since $\lambda_{2}^{K}$ is induced from $B Z$ it also vanishes on the class not containing $b$, and $\lambda_{2}^{K}(b)=2$ because there are two coset representatives of $Z B$ in $K$ which conjugate $b$ into $B Z$, namely 1 and $h^{2^{n-2}}$. Thus $\lambda_{1}^{K}$ and $\lambda_{2}^{K}$ agree outside $H$ and the difference $\lambda_{1}^{K}-\lambda_{2}^{K}$ vanishes outside $H$ so we have completed the proof for the dihedral case.

In the semidihedral case $b^{-1} h b=h^{-1} z$ and $h^{-1} b h=b h^{2} z$. The two conjugacy classes of the dihedral group lying outside $H$ are closed under multiplication by $z$ so one can see that they are also conjugacy classes of the semidihedral group. The proof in this case is essentially the same as for the dihedral case.

Finally we consider $K$ of type $M_{n+1}(2)$. Here $b^{-1} h b=h z$ and $h^{-1} b h=b z$. Outside of $H$ there are $2^{n-1}$ conjugacy classes each with two elements, one being $z$ times the other. Both characters $\lambda_{1}^{K}$ and $\lambda_{2}^{K}$ can have nonzero value only on the class consisting of $b$ and $b z$ since only this class intersects the subgroups $B$ and $B Z$. Now $\lambda_{1}^{K}(b)=|C(b)| /|b|=2^{n} / 2=2^{n-1}$ because $C(b)$ is the subgroup generated by $h^{2}$ and $b$ of order $2^{n}$. Since $B Z$ is a normal subgroup $K, \lambda_{2}^{K}$ has kernel $B Z$. Therefore $\lambda_{2}^{K}(b)=[K: B Z]=2^{n-1}$. Again we have shown $\lambda_{1}^{K}-\lambda_{2}^{K}$ vanishes outside $H$ which completes the proof of the theorem.

The quaternion group of order 8 illustrates the necessity of the denominator 2 in the theorem. The irreducible character of degree 2 is one-half the difference of the characters induced from the principal characters on the identity and the center of order 2.

4. A special case. There is a somewhat different version of Theorem $\mathbf{A}$ that has appeared in Ford [3, p. 627, Lemma 1] and Yamada [9, p. 299, Proposition 1] and probably orginates in Witt [8]. Comparing the two versions leads to the following situation. Let $H$ and $A$ be cyclic groups of order $p^{n}$ and $p^{a}$ respectively, with $a \leq n-1$ if $p$ is odd and $a \leq n-2$ if $p=2$. There is a subgroup of the automorphism group of $H$ which is isomorphic to $A$, and we let this define a faithful action of $A$ on $H$. This makes $H A$ into a semidirect product. If $p=2$ we assume that $A$ fixes the element of order 4 in $H$. This excludes generalized quaternion, dihedral, and semidihedral groups.

The center of $H A$ is the subgroup $Z$ of $H$ of index $p^{a}$. Let $\mu$ be a faithful linear character on $H$. Let $\chi=\mu^{H A}$. One can show that $[Q(\mu): Q(\chi)]=p^{a}$. There 
must exist a linear character $\lambda$ inducing $\chi$ with $Q(\lambda)=Q(\chi)$. The following works: choose $\lambda$ a character on $Z A$ which is faithful on $Z$ with kernel $A$. To see that $\lambda^{H A}=\chi$ apply the Mackey subgroup theorem

$$
\left.\mu^{H A}\right|_{Z A}=\left(\left.\mu\right|_{Z A \cap H}\right)^{Z A}=\left(\left.\mu\right|_{Z}\right)^{Z A} .
$$

Now calculate the inner product

$$
\left(\mu^{H A}, \lambda^{H A}\right)=\left(\left.\mu^{H A}\right|_{Z A}, \lambda\right)_{Z A}=\left(\left(\left.\mu\right|_{Z}\right)^{Z A}, \lambda\right)_{Z A}=\left(\left.\mu\right|_{Z},\left.\lambda\right|_{Z}\right)_{Z}=1 .
$$

Since $\mu^{H A}$ is irreducible and has the same degree as $\lambda^{H A}$ the two are equal.

Starting with character $\chi$, repeated application of Theorem A would yield character $\lambda$. However applying this different version could yield $\mu$. We have shown here how to make the transition between the two.

I wish to express thanks to Gerald Janusz for some helpful conversations during the preparation of this paper.

Addendum. The main theorem has the following corollaries.

THEOREM. Each irreducible rational representation of a finite p-group is induced from the irreducible faithful rational representation of degree $p-1$ on a section of order $p$.

In the case $p=2$ this reduces to the following result.

THEOREM. Each irreducible rational representation of a finite 2-group is induced from the faithful linear character on a section of order 2 .

To prove the first theorem, we have shown that the character of such a representation can be written as the difference $\lambda_{1}^{G}-\lambda_{2}^{G}$ where $\lambda_{1}$ and $\lambda_{2}$ are the principal characters on subgroups $G_{1}$ and $G_{2}$ respectively with $G_{1}$ a subgroup of index $p$ in $G_{2}$. By first inducing to $G_{2}$ and then to $G$ this can be written as $\left(\lambda_{1}^{G_{2}}-\lambda_{2}\right)^{G}$. Now $\lambda_{1}^{G_{2}}$ is the regular character of degree $p$ and $\lambda_{2}$ the principal character on the quotient $G_{2} / G_{1}$. This difference is the character of the faithful irreducible rational representation of degree $p-1$ on $G_{2} / G_{2}$ which is a cyclic group of order $p$. This proves the assertion.

As a special case of the second theorem we note that the faithful rational 4dimensional representation of the quaternion group of order 8 is induced from the faithful linear character on the center of order 2 .

ADDED IN PROOF. The method of proof used here is also used by John R. Rasmussen in Rationally represented characters and permutation characters of nilpotent groups, J. Algebra 29 (1974), 504-509.

\section{REFERENCES}

1. C. Curtis and I. Reiner, The representation theory of finite groups and associative algebras, Interscience, New York, 1962.

2. W. Feit, Characters of finite groups, Benjamin, New York, 1967.

3. C. Ford, Some results on the Schur index of a representation of a finite group, Canad. J. Math. 22 (1970), 626-640.

4. D. Gorenstein, Finite groups, Chelsea, New York, 1980.

5. T. Y. Lam, Artin exponent of finite groups, J. Algebra 9 (1968), 94-119.

6. G. Segal, Permutation representation of finite p-groups, Quart. J. Math. Oxford (2) 23 (1972), $375-381$. 
7. L. Solomon, The representation of finite groups in algebraic number fields, J. Math. Soc. Japan 13 (1961), 144-164.

8. E. Witt, Die Algebraische Strucktur des Gruppenringes einer endlichen Gruppen über einem Zahlkörper, J. Reine Angew. Math. 190 (1952), 231-245.

9. T. Yamada, Characterization of simple components of the group algebras over the p-adic number field, J. Math. Soc. Japan 23 (1971), 295-310.

DePARTMENT OF MATHEMATICS AND COMPUTER SCIENCE, SAINT LOUIS UNIVERSITY, ST. LOUIS, MISSOURI 63103 